Note: This is an unpublished manuscript. We welcome any feedback or comments.

\title{
Title: Consensus-based guidance for conducting and reporting multi-analyst studies
}

Authors' names: Balazs Aczel ${ }^{1 *}$, Barnabas Szaszi ${ }^{1}$, Gustav Nilsonne ${ }^{2,3}$, Olmo R van den Akker $^{4}$, Casper J Albers ${ }^{5}$, Marcel A L M van Assen ${ }^{4,6}$, Jojanneke A Bastiaansen ${ }^{7,8}$, Dan Benjamin $^{9,10}$, Udo Boehm ${ }^{11}$, Rotem Botvinik-Nezer ${ }^{12}$, Laura F Bringmann ${ }^{5}$, Niko A Busch ${ }^{13}$, Emmanuel Caruyer ${ }^{14}$, Andrea M Cataldo ${ }^{15,16}$, Nelson Cowan ${ }^{17}$, Andrew Delios ${ }^{18}$, Noah N N van Dongen ${ }^{11}$, Chris Donkin ${ }^{19}$, Johnny B van Doorn ${ }^{11}$, Anna Dreber ${ }^{20,21}$, Gilles Dutilh ${ }^{22}$, Gary F Egan ${ }^{23}$, Morton Ann Gernsbacher ${ }^{24}$, Rink Hoekstra ${ }^{5}$, Sabine Hoffmann ${ }^{25}$, Felix Holzmeister $^{21}$, Juergen Huber ${ }^{21}$, Magnus Johannesson ${ }^{20}$, Kai J Jonas ${ }^{26}$, Alexander T Kindel ${ }^{27}$, Michael Kirchler ${ }^{21}$, Yoram K Kunkels ${ }^{7}$, D Stephen Lindsay ${ }^{28}$, Jean-Francois Mangin ${ }^{29,30}$, Dora Matzke $^{11}$, Marcus R Munafò ${ }^{31}$, Ben R Newell ${ }^{19}$, Brian A Nosek ${ }^{32,33}$, Russell A Poldrack ${ }^{34}$, Don van Ravenzwaaij ${ }^{5}$, Jörg Rieskamp ${ }^{35}$, Matthew J Salganik ${ }^{27}$, Alexandra Sarafoglou ${ }^{11}$, Tom Schonberg $^{36}$, Martin Schweinsberg ${ }^{37}$, David Shanks ${ }^{38}$, Raphael Silberzahn ${ }^{39}$, Daniel J Simons $^{40}$, Barbara A Spellman ${ }^{33}$, Samuel St-Jean ${ }^{41,42}$, Jeffrey J Starns ${ }^{43}$, Eric L Uhlmann ${ }^{44}$, Jelte Wicherts ${ }^{4}$, Eric-Jan Wagenmakers ${ }^{11}$

Affiliations: ${ }^{1}$ ELTE, Eotvos Lorand University, Budapest, Hungary, ${ }^{2}$ Karolinska Institutet, Stockholm, Sweden, ${ }^{3}$ Stockholm University, Stockholm, Sweden, ${ }^{4}$ Tilburg University, Tilburg, The Netherlands, ${ }^{5}$ University of Groningen, Groningen, The Netherlands, ${ }^{6}$ Utrecht University, Utrecht, The Netherlands, ${ }^{7}$ University of Groningen, University Medical Center Groningen, Groningen, The Netherlands, ${ }^{8}$ Friesland Mental Health Care Services, Leeuwarden, The Netherlands, ${ }^{9}$ University of California Los Angeles, Los Angeles, CA, USA, ${ }^{10}$ National Bureau of Economic Research, Cambridge, MA, USA, ${ }^{11}$ University of Amsterdam, Amsterdam, The Netherlands, ${ }^{12}$ Dartmouth College, Hanover, NH, USA, ${ }^{13}$ University of Münster, Münster, Germany, ${ }^{14}$ University of Rennes, CNRS, Inria, Inserm, Rennes, France, ${ }^{15}$ McLean Hospital, Belmont, MA, USA, ${ }^{16}$ Harvard Medical School, Boston, MA, USA, ${ }^{17}$ Department of Psychological Sciences, University of Missouri, MO, USA, ${ }^{18}$ National University of Singapore, Singapore, ${ }^{19}$ University of New South Wales, Sydney, Australia, ${ }^{20}$ Stockholm School of Economics, Stockholm, Sweden, ${ }^{21}$ University of Innsbruck, Innsbruck, Austria, ${ }^{22}$ University Hospital Basel, Basel, Switzerland, ${ }^{23}$ Monash University, Melbourne, Victoria, Australia, ${ }^{24}$ University of Wisconsin-Madison, Madison, WI, USA, ${ }^{25}$ Ludwig-Maximilians-University, Munich, Germany, ${ }^{26}$ Maastricht University, Maastricht, The Netherlands, ${ }^{27}$ Princeton University, Princeton, NJ, USA, ${ }^{28}$ University of Victoria, Victoria, Canada, ${ }^{29}$ Université Paris-Saclay, Paris, France, ${ }^{30}$ Neurospin, CEA, France, ${ }^{31}$ University of Bristol, Bristol, UK, ${ }^{32}$ Center for Open Science, USA, ${ }^{33}$ University of Virginia, Charlottesville, USA, ${ }^{34}$ Stanford University, Stanford, USA, ${ }^{35}$ University of Basel, Basel, Switzerland, ${ }^{36} \mathrm{Tel}$ Aviv University, Tel Aviv, Israel, ${ }^{37}$ ESMT Berlin, Germany, ${ }^{38}$ University College London, London, UK, ${ }^{39}$ University of Sussex, Brighton, UK, ${ }^{40}$ University of Illinois at Urbana-Champaign, USA, ${ }^{41}$ University of Alberta, Edmonton, Canada, ${ }^{42}$ Lund University, Lund, Sweden, ${ }^{43}$ University of Massachusetts Amherst, USA, ${ }^{44}$ INSEAD, Singapore

${ }^{*}$ Correspondence: B Aczel aczel.balazs@ppk.elte.hu 


\begin{abstract}
The analysis of most clinical and other empirical datasets allows for alternative strategies, therefore, reported findings of a single analysis may not represent the full range of possible and justifiable estimates derivable from the same data. The multi-analyst approach is a method to systematically assess whether the reported results and conclusions are dependent on the chosen analytical strategy. We present consensus-based guidance and checklist for conducting and reporting multi-analyst studies, in which many analysts independently analyse the same dataset. We discuss why broader adoption of the multi-analyst approach will strengthen the robustness of results and conclusions in clinical and basic sciences.
\end{abstract}

\title{
Introduction
}

Empirical results typically hinge on analytical choices made by a single data analyst or team of authors, with limited independent, external input. This makes it uncertain whether the reported conclusions are robust to justifiable alternative analytical strategies (Fig.1). Empirical investigations require many analytical decisions, and the theories motivating the research rarely imposes strong restrictions on how the data should be preprocessed and modelled. Reported estimates of effects may not represent the full range of possible and justifiable estimates derivable from the same data. This analytical variability may impact assessments of the evidence and the associated conclusions in basic and applied research.
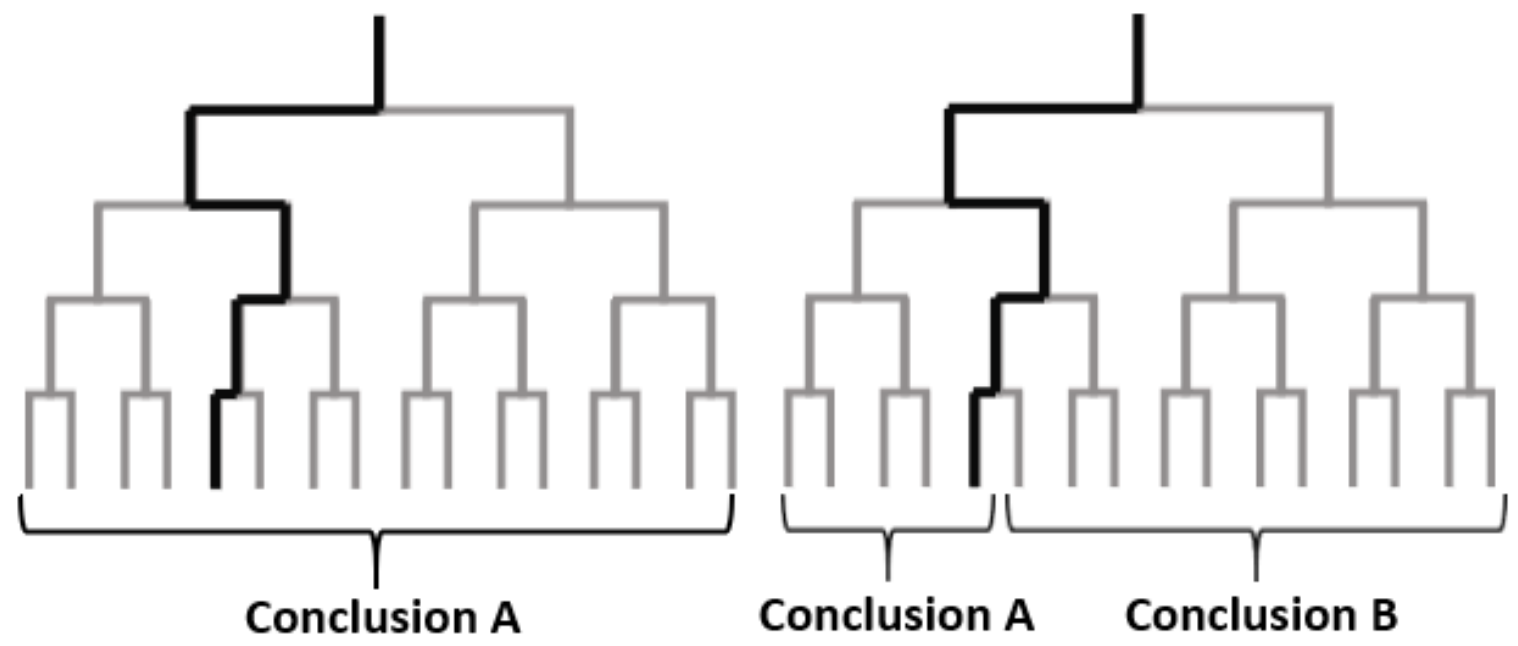

Fig.1 Example of a reported sequence of analysis choices (black line, leading to conclusion A) shown as a subset of alternative plausible analysis paths (grey lines). In the left panel, all plausible paths support conclusion $\mathrm{A}$; in the right panel, most plausible paths support conclusion B. This illustrates that without testing alternative paths, it remains unknown whether the conclusion is robust to justifiable alternative analytical strategies.

The analytical variability may be particularly high for datasets that were not initially recorded for research purposes, since we typically only have poor knowledge on the exact measurement procedures and data generation mechanisms. The increasing availability of large routinely collected datasets, for instance through administrative claims and electronic health records, raises hopes and promises of "real-world" evidence and personalized treatment regimes. Yet these hopes and promises are accompanied by a growing realization 
that the potential analytical variability is much greater in the analysis of these datasets than in the analysis of data from clinical trials or non-randomized studies with prospective data collection.

As an example of analytical variability in medical database research, the journal Surgery published two articles $(1,2)$ a few months apart that used the same dataset to investigate the same question: Does the use of a retrieval bag during laparoscopic appendectomy reduce surgical site infections? Two reasonable, but different, analyses were applied (with notable differences in analytical choices including inclusion and exclusion criteria, outcome measures, sample sizes, and covariates). As a result of the different analytical choices, the two articles reached opposite conclusions, one finding that the use of a retrieval bag reduced infections, and the other that it did not (3). This and other medical examples (4-6) illustrate how independent analysis of the same data (in this case, unplanned) can reach different, yet justifiable, conclusions.

The analytical robustness of results and conclusions can be studied by evaluating many distinct analysis options simultaneously (e.g., vibration of effects (7) or multiverse analysis (8)) or by involving multiple analysts who independently analyse the same data (9-18). Rather than exhaustively evaluating all plausible analyses, the multi-analyst method examines analytical choices that are deemed most appropriate by independent analysts.

Botvinik-Nezer et al. (13), for example, asked 70 teams to test the same hypotheses using the same functional magnetic resonance imaging dataset. They found that no two teams followed the same data preprocessing steps or analysis strategies, resulting in substantial variability in their conclusions. This and other initiatives (9-18) demonstrate that the multi-analyst approach is able to explore how research findings vary depending on the applied analytic strategy.

Although the multi-analyst approach is new to many researchers, it has been in use since the 19th century. A prominent example is the cuneiform competition (19), which may be viewed as a precursor to the modern multi-analyst method. In 1857, the Royal Asian Society asked four scholars to independently translate a previously unseen inscription to verify that the ancient Assyrian language had been deciphered correctly. The almost perfect overlap between the solutions indicated that "they have Truth for their basis" (p4 19).

The central idea from this cuneiform competition can be applied to 21 st century data analysis with several benefits (Box 1). With even a few co-analysts, the multi-analyst approach can be informative about the analytic robustness of results and conclusions. When the results of independent data analyses converge, more confidence in the conclusions is warranted. When the results diverge, confidence appropriately falters, and scientists can examine the reasons for these discrepancies. With enough co-analysts, it is possible to estimate the variability among analysis strategies and identify factors explaining this variability. 
Box 1. Benefits of the Multi-Analyst Approach

\section{Benefits of the Multi-Analyst Approach}

- Converging conclusions increase confidence in the analytical robustness of a finding

- Diverging conclusions decrease confidence in the analytical robustness of a finding and prompt an examination of the reasons for the divergence

- Identifies a key source of uncertainty and variability, namely the extent to which the results and conclusions depend on the analytic preferences of the analyst

- With analysts from multiple disciplines, the approach stimulates cross-pollination of analysis strategies that otherwise might remain isolated within research subcultures

- Diminishes or eliminates the analyst's potential preference toward the hypotheses, because no individual analysis is likely to determine the conclusions

The multi-analyst approach is still rarely used, but we argue that many disciplines could benefit from its broader adoption. To help researchers overcome practical challenges, we provide consensus-based guidance and checklist to help researchers surmount the practical challenges of preparing, conducting, and reporting multi-analyst studies.

\section{Methods}

To develop this guidance, we recruited a panel of 50 methodology experts who followed a preregistered 'reactive-Delphi' expert consensus procedure.

\section{Preparation}

\section{Preregistering the project}

Before the start of the project, on 11 November 2020, a research plan was compiled and uploaded to a time-stamped repository at https://osf.io/dgrua. During the project, we followed the preregistered plan in all respects except implementing slight changes in the wording of the survey questions to improve comprehension. Although we declared that we would share the $\mathrm{R}$ code and codebook of our analyses, the project did not require us to conduct analyses in $\mathrm{R}$.

\section{Creating the initial Multi-Analyst Guidance draft}

Before the expert consensus process, the first three authors and the last author (henceforth: proposers) created an initial multi-analyst guidance draft after brainstorming and reviewing all the previously published multi-analyst-type projects they were aware of (9-18). This initial document is available here: https://osf.io/kv8jt/

\section{Recruiting experts}

The proposers contacted 81 experts to join the project. The contacted experts included all the organisers of the previous known multi-analyst projects at the time (9-18), as well as experts 
on research methodology. Of the 81 experts, 50 accepted the invitation and participated in the expert consensus procedure (their names are available at https://osf.io/fwqvp/).

\section{Preparatory rounds}

Upon joining the project, the experts received a link to the preparatory online survey (available at https://osf.io/kv8jt/) which included the initial Multi-Analyst Guidance draft where they had the option to comment on each of the items and the overall content of the guidance.

Based on the feedback received from the preparatory online survey, the proposers updated and revised the initial Multi-Analyst Guidance. This updated document was uploaded to an online shared document and was sent out to the experts who had the option to edit and comment on the content. Again, based on feedback, the proposers revised the content of the document, and this new version was included in the expert consensus survey.

\section{Consensus survey}

The expert consensus questionnaire was sent out individually to each expert first on 8 February 2021 in the following Qualtrics survey available at https://osf.io/wrpnq/.

The survey contained the 10 recommended practices grouped into the following 5 stages: Recruiting Co-Analysts; Providing the Dataset, Research Questions, and Research Tasks; Conducting the Independent Analyses; Processing the Results; and Reporting the Methods and Results. The respondents were asked to rate each of the 10 recommended practices on a 9-point Likert-type scale ('I agree with the content and wording of this guidance section' ranging from "1-Disagree" to "9-Agree"). Following each section, the respondents could leave comments regarding the given item.

The preregistration indicated consensus on the given item, if the interquartile range of the corresponding ratings was 2 or smaller; it defined support for an item if the median of its corresponding ratings was 6 or higher. We received completed ratings from 48 experts and found consensus for each of the recommended practices in our first round. That is, all of the items have been found to be eligible to enter the guidance with consensual support. The summary table of the results is available at https://osf.io/qc7a8/.

\section{Finalising the manuscript}

The proposers drafted the manuscript and supplements. All texts and materials were sent to the expert panel members. Each contributor was encouraged to provide feedback on the manuscript, the report, and the suggested final version of the guidance. After all discussions, minor wording changes were implemented, as documented at https://osf.io/e39j4/. No contributor objected to the content and form of the submitted materials and all approved the final item list.

\section{Multi-analyst Guidance}


The final guidance includes 10 Recommended Practices (Table 1) concerning the 5 main stages of multi-analyst studies. To further assist researchers in documenting multi-analyst projects, we also provide a modifiable Reporting Template (Appendix 1), as well as a Reporting Checklist (Appendix 2) developed from our guide.

Table 1

Recommended Practices for the Main Stages of the Multi-Analyst Method

Stage Recommended Practices

Recruiting

Co-analysts

Providing the

Dataset,

Research

Questions, and Research

Tasks

Conducting

the

Independent

Analyses

Processing the Results
1. Determine a minimum target number of co-analysts and outline clear eligibility criteria before recruiting co-analysts. We recommend that the final report justifies why these choices are adequate to achieve the study goals.

2. When recruiting co-analysts, inform them about (a) their tasks and responsibilities; (b) the project code of conduct (e.g., confidentiality/ non-disclosure agreements); (c) the plans for publishing the research report and presenting the data, analyses, and conclusion; (d) the conditions for an analysis to be included or excluded from the study; (e) whether their names will be publicly linked to the analyses; (f) the co-analysts' rights to update or revise their analyses; $(\mathrm{g})$ the project time schedule; and (h) the nature and criteria of compensation (e.g., authorship).

3. Provide the datasets accompanied with a codebook that contains a comprehensive explanation of the variables and the datafile structure.

4. Ensure that co-analysts understand any restrictions on the use of the data, including issues of ethics, privacy, confidentiality, or ownership.

5. Provide the research questions (and potential theoretically derived hypotheses that should be tested) without communicating the lead team's preferred analysis choices or expectations about the conclusions.

6. To ensure independence, we recommend that co-analysts should not communicate with each other about their analyses until after all initial reports have been submitted. In general, it should be clearly explained why and at what stage co-analysts are allowed to communicate about the analyses (e.g., to detect errors or call attention to outlying data points).

7. Require co-analysts to share with the lead team their results, the analysis code with explanatory comments (or a detailed description of their point-and-click analyses), their conclusions, and an explanation of how their conclusions follow from their results.

8. The lead team makes the commented code, results, and conclusions of all non-withdrawn analyses publicly available before or at the same time as submitting the research report. 
Reporting
the Methods
and Results
9. The lead team should report the multi-analyst process of the study, including (a) the justification for the number of co-analysts; (b) the eligibility criteria and recruitment of co-analysts; (c) how co-analysts were given the data sets and research questions; (d) how the independence of analyses was ensured; (e) the numbers of and reasons for withdrawals and omissions of analyses; (f) whether the lead team conducted an independent analysis; ( $\mathrm{g}$ ) how the results were processed; (h) the summary of the results of co-analysts; (i) and the limitations and potential biases of the study.

10. Data management should follow the FAIR principles (20), and the research report should be transparent about access to the data and code for all analyses (21).

\section{Practical Considerations}

In addition to the Multi-analyst Guidance and Checklist, we provide practical considerations that can support the organization and execution of multi-analyst projects. This section contains various clarifications, recommendations, practical tools, and optional extensions, covering the five main stages of a multi-analyst project: Recruiting Co-analysts; Providing the Dataset, Research Questions, and Research Tasks; Conducting the Independent Analyses; Processing the Results; and Reporting the Methods and Results.

\section{$\underline{\text { Recruiting Co-analysts }}$}

\section{Choosing co-analysts}

The term co-analyst refers to one researcher or team of researchers working together in a multi-analyst project. Researchers can collaborate on the analyses, but if they do, we recommend that they submit the analyses as one co-analyst team, in order to ensure the independence of the analyses. Researchers from the same lab or close collaborators should be able to submit separate reports in the multi-analyst project as long as they do not discuss their analyses outside of their respective teams until the project rules allow that. The lead team may conduct an analysis themselves depending on the study goals and the design of the project (e.g., to set a performance baseline for comparing submitted models). Alternatively, the lead team may choose not conduct an analysis themselves; in any case, they are expected to be transparent about their level of involvement as well as the timing (e.g., whether they conducted their analyses with or without knowing the results of the crowd of analysts).

Researchers should carefully consider both the breadth and depth of statistical and research-area expertise required for their project and should justify their choices about the required qualifications, skills, and credentials for analysts in the project. If the aim of the study is to explore what factors influence researchers' analytical choices, then it can be 
useful to seek "natural variation" (representativeness) within an expert community or to maximize diversity of the co-analysts along the dimensions where they might differ the most in their choices (e.g., experience, background, discipline, interest in the findings, intellectual allegiance to different theories, paradigmatic viewpoints).

\section{Deciding on the number of co-analysts}

To decide on the desired number of co-analysts, one has to consider which of the two main purposes of the multi-analyst method applies to the given project:

\section{(A) Checking the robustness of the conclusions}

The aim here is solely to check whether different analysts obtain the same conclusions. Confidence in the stability of the conclusions decreases with divergent results and increases with convergent results. Most projects can achieve this aim by recruiting only one or a handful of additional analysts.

(B) Assessing the variability of the analyses

Those who wish to estimate the variability among the different analytical strategies often need to satisfy stricter demands. For example, studies that aim to assess how much the results vary among the analysts will require a larger number of co-analysts. When determining the number of co-analysts in such cases, the same factors need to be taken into consideration as in standard sample size estimation methods.

\section{$\underline{\text { Recruiting co-analysts }}$}

Depending on the specific goal of the research, the recruitment of co-analysts can happen in several ways. Co-analysts can be recruited before or after obtaining the dataset. With stricter eligibility criteria, co-analysts can be invited individually from among topic experts or statistical experts. Follow-up open invitations can ask experts to suggest others to be invited. Alternatively, the lead team can open the opportunity to anyone to join the project as a co-analyst within the expert community (e.g., in professional society mailing lists and on social media), where expertise can be defined as the topic requires it.

It is important to note that whenever the co-authors' behavior is the subject of the study then they should be regarded similarly to human participants respecting ethical and data protection regulations.

\section{Providing the Dataset, Research Questions, and Research Tasks}

\section{Providing the dataset}

The lead team can invite the co-analysts to conduct data preprocessing (in addition to the main analysis). If the lead team decides to conduct the preprocessing themselves, showing their preprocessing methods can be informative to the co-analysts, but also has the 
potential to influence them if the preprocessing reflects some preference of methods or expectations of outcomes.

Before providing the dataset, the lead team should ensure that data management will comply with legal (e.g., the General Data Protection Regulation (GDPR) in the European Union) and ethical regulations applying to all teams (see 22). If the dataset contains personal information, a version should be provided where data can no longer be related to an individual. An alternative is to provide a simulated dataset and ask the co-analysts to provide code to analyse the data $(23,24)$. The lead team can then run the code on the actual data.

It is important that the co-analysts understand not just the available dataset but also any ancillary information that might affect their analyses (e.g., prior exclusion of outliers or handling of missing data in the blinded dataset). Providing a codebook that is accessible and understandable for researchers with different backgrounds is essential (25).

\section{Providing the task}

The multi-analyst approach can leave the operationalization of the research question to the co-analysts so that they can translate the theoretical question into the measurement. Taking this approach can reveal the operational variations of a question, but it can also make it difficult to compare the statistical results.

Requesting results on a standardized metric makes it easier to compare results between co-analysts. However, this will likely bias the analysis strategies towards using methods that easily provide such a metric. [Practical tool: instructions for reporting an effect estimate: https://osf.io/kr2g9/]

It can be useful to ask the co-analysts to keep a record of any code, derivatives etc. that were part of the analysis, at least until the manuscript is submitted and all relevant materials are (publicly) shared.

As an extension, the co-analysts can be asked to record considered but rejected analysis choices and the reasoning behind their choices (e.g., by commented code, log books, or dedicated solutions such as DataExplained (26)). These logs can reflect where and why co-analysts diverge in their choices.

Robustness, or multiverse analyses (in the sense that each team is free to provide a series of outcomes instead of a single one) can also be part of the task of the co-analysts so that multiple analyses are conducted under alternative data analysis preprocessing choices.

\section{$\underline{\text { Communication with co-analysts }}$}

In projects with many co-analysts, keeping contact via a dedicated email address and automating some of the messages (e.g., automated emails when teams finished a stage in the process) can help streamline the communication and make the process less prone to human 
errors. For co-analyst teams with multiple members, it can be helpful for each team to nominate one member as the representative for communications.

If further information is provided to a co-analyst following specific questions, it can be useful to make sure the same information is provided to all teams, for example via a Q\&A section of the project website, hosting weekly office hours where participants could ask questions, or via periodic email with updates.

\section{Conducting the Independent Analyses}

\section{Preregistering the process}

We can distinguish meta- and specific preregistrations. Meta-preregistrations concern the plan of the whole multi-analyst project. It is good practice for the lead team to preregister how they would process, handle, and report the results of the co-analysts in order to prevent result-driven biases. This can be done in the form of a Registered Report at journals that invite such submissions (27). Any metascientific questions, such as randomisation of co-analysts to different conditions with variations in instructions or data, or covariates of interest for studying associations to analytic variability, should be specified.

Specific preregistrations concern the analysis plans of the co-analysts. Requiring co-analysts to prepare a specific preregistration for each analysis can be a strategy to prevent overfitting and undisclosed flexibility. It makes sense to require it from either all or none of the teams in order to maintain equal treatment among them (unless the effect of preregistration is a focus of the study).

Requiring specific preregistrations may be misaligned with the goals of the project when the aim is to explore how the analytic choices are formed during the analyses, independent of initial plans. Under such circumstances, requiring specific preregistrations may be counterproductive. Nevertheless, the lead team can record their meta-preregistration that lays down the details of the multi-analyst project.

There are alternative solutions to prevent researchers from being biased by their data and results. For example, co-analysts could be provided with blinded datasets $(14,16,28)$, simulated datasets (24), or with a subset of the data (e.g., 11).

\section{Processing the Results}

\section{Collecting the results}

To facilitate summarizing the co-analysts' methods, results, and conclusions, the lead team can collect results through provided templates or survey forms that can structure analysts' reports. It is practical to ask the co-analysts at this stage to acknowledge that they did not communicate or cooperate with other co-analysts regarding the analysis in the project. It can also be helpful for the lead team if the co-analysts explain how their conclusions were derived from the results. In case preregistration was employed for any 
analyses, the template can also collect any deviations from the preregistered plan for inclusion in an online supplement.

To collect analytic code, it may be useful to require a container image $(29,30)$ or a portable version of the code that handles issues like software package availability (31).

\section{Validating the results}

The lead team is recommended to ensure that each analyst's codes/procedures reproduce that analyst's submitted results. Computational reproducibility can be ascertained by running the code or repeating the analytic process by the lead team, but independent experts or the other co-analysts can also be invited to undertake this task $(32,33)$.

The project can leverage the crowd by asking co-analysts to review others' analyses, or the lead team can employ external statistical experts to assess analyses and detect major errors. The lead team can decide to omit analyses with major errors. In that case, the reasons for omission should be documented, and for transparency, the results of the omitted analyses should be included in an online supplement.

After all the analyses have been submitted and validated, the co-analysts could have the option in certain projects to inspect the work of the other analysts and freely withdraw their own analyses. This can be appropriate if seeing other analyses makes them aware of major mistakes or shortcomings in their analytic procedures. A potential bias in this process is that co-analysts might lose confidence in their analyses after seeing other, more senior, or more expert co-analysts' work. One way to decrease this potential bias is to follow a multi-stage process: after the first round of analyses is submitted, co-analysts could be allowed to see each other's analysis steps/code without knowing the identity of the co-analyst or the results of their analysis. It is the lead team's decision whether they allow co-analysts to correct or update their analyses after an external analyst or the co-analysts themselves find issues in their analyses.

Importantly, it is a minimum expectation that from the start of the project, the co-analysts should know about the conditions for their analyses to be included in, or omitted from, the study. All withdrawals, omissions, and updates of the results should be transparent in subsequent publications, for example in the supplementary materials.

\section{Reporting the Methods and Results}

Recording contributorship

Using CRediT taxonomy can transparently record organizers' and co-analysts' contributions to the study. Practical tools (e.g., tenzing 34) can make this task easier.

Co-analysts can be invited to be co-authors and/or to be compensated for their contribution in other ways (e.g., prizes, honorariums). Expectations for contribution and authorship should be communicated clearly at the outset. 
Presenting the methods and results

Beyond a descriptive presentation of results in a table or graph, the reporting of the results of multi-analyst projects is not straightforward and remains an open area of research. If the main purpose is to estimate variability of analyses, it is interesting to investigate and report factors that might influence variability in the chosen analytic approaches and in the results obtained by these analytical approaches. If, on the other hand, the main purpose is to investigate the robustness of conclusions by assessing the degree to which different analysts obtain the same results, it is advisable to focus more on methods that produce only a single answer to the research question of interest.

The analytical approach of each co-analyst can be divided into discrete choices concerning, for instance, data preprocessing steps and decisions in model specification. If it is possible to recombine the individual choices (which will not always be the case as certain data preprocessing steps or method choices may only make sense if the aim is to fit a certain class of models), it may be worthwhile to create a larger set of possible analytical approaches that is made up of all possible combinations. In this case, the descriptive results of the multi-analyst project can be combined with a multiverse type approach (e.g., vibration of effects 7 , multiverse analysis 8 , or specification curve 35) to quantify and compare the variability in results that can be explained by the different analytical choices $(36,37)$. Additionally, this larger set of possible combinations can be helpful to present the results in an interactive user interface in which readers can explore how the results change as a function of certain analytical choices $(37,38)$. Finally, dividing the co-analysts' analytical approaches into individual choices may ultimately help in providing a unique answer to the research question of interest while accounting for the uncertainty in the choice of the analytical approach. While there are so far no approaches that would allow the derivation of a unique result that integrates all uncertain decisions, it may be a promising area of research to extend Bayesian approaches that account for model uncertainty (39) and measurement error (40).

To support the reporting of Multi-Analyst projects, we provide a freely modifiable Reporting Template available from here: https://osf.io/h9mgy/

\section{Limitations}

The present work does not cover all aspects of multi-analyst projects. For instance, the multi-analyst approach outlined here entails the independent analysis of one or more datasets, but it should be acknowledged that other crowdsourced analysis approaches might not require such independence of the analyses. Also, we emphasise that this consensus-based guidance is a first step towards the broader adoption of the multi-analyst approach in empirical research; we hope and expect that our recommendations will be developed further.

\section{Conclusions}

This guidance document aims to facilitate adoption of the multi-analyst approach in clinical and basic research. Although the multi-analyst approach is at an incipient stage of adoption, we believe that the scientific benefits greatly outweigh the extra logistics required, 
especially for projects with high relevance for clinical practice and policy making. The approach should have key relevance for meta-analyses, if choosing different analytical strategies on the same dataset may lead to conflicting results, confusing practitioners and the scientific community. The multi-analyst approach allows a systematic exploration of the analytical space to assess whether the reported results and conclusions are dependent on the chosen analytical strategy, ultimately improving the reliability and credibility of research findings.

\section{References}

1. Fields AC, Lu P, Palenzuela DL, Bleday R, Goldberg JE, Irani J, Davids JS, Melnitchouk N. Does retrieval bag use during laparoscopic appendectomy reduce postoperative infection? Surgery. 2019;165(5):953-7.

2. Turner SA, Jung HS, Scarborough JE. Utilization of a specimen retrieval bag during laparoscopic appendectomy for both uncomplicated and complicated appendicitis is not associated with a decrease in postoperative surgical site infection rates. Surgery. 2019;165(6):1199-202.

3. Childers CP, Maggard-Gibbons M. Same Data, Opposite Results?: A Call to Improve Surgical Database Research. JAMA Surg. 2021;156(3):219-20.

4. de Vries M, Witteman CLM, Holland RW, Dijksterhuis A. The Unconscious Thought Effect in Clinical Decision Making: An Example in Diagnosis. Med Decis Making. 2010;30(5):578.

5. Jivanji D, Mangosing M, Mahoney SP, Castro G, Zevallos J, Lozano J. Association Between Marijuana Use and Cardiovascular Disease in US Adults. Cureus. 2020 Dec 3;12(12).

6. Shah S, Patel S, Paulraj S, Chaudhuri D. Association of Marijuana Use and Cardiovascular Disease: A Behavioral Risk Factor Surveillance System Data Analysis of 133,706 US Adults. Am J Med. 2021 May 1;134(5):614-620.e1.

7. Patel CJ, Burford B, Ioannidis JP. Assessment of vibration of effects due to model specification can demonstrate the instability of observational associations. J Clin Epidemiol. 2015;68(9):1046-58.

8. Steegen S, Tuerlinckx F, Gelman A, Vanpaemel W. Increasing Transparency Through a Multiverse Analysis. Perspect Psychol Sci. 2016 Sep 1;11(5):702-12.

9. Bastiaansen JA, Kunkels YK, Blaauw FJ, Boker SM, Ceulemans E, Chen M, Chow S-M, de Jonge P, Emerencia AC, Epskamp S, Fisher AJ, Hamaker EL, Kuppens P, Lutz W, Meyer MJ, Moulder R, Oravecz Z, Riese H, Rubel J, Ryan O, Servaas MN, Sjobeck G, Snippe E, Trull TJ, Tschacher W, van der Veen DC, Wichers M, Wood PK, Woods WC, Wright AGC, Albers CJ, Bringmann LF. Time to get personal? The impact of researchers choices on the selection of treatment targets using the experience sampling methodology. J Psychosom Res. 2020 Oct $1 ; 137: 110211$.

10. Dongen NNN van, Doorn JB van, Gronau QF, Ravenzwaaij D van, Hoekstra R, Haucke MN, Lakens D, Hennig C, Morey RD, Homer S, Gelman A, Sprenger J, Wagenmakers E-J. Multiple Perspectives on Inference for Two Simple Statistical Scenarios. Am Stat. 2019 Mar 29;73(sup1):328-39.

11. Salganik MJ, Lundberg I, Kindel AT, Ahearn CE, Al-Ghoneim K, Almaatouq A, Altschul DM, Brand JE, Carnegie NB, Compton RJ, Datta D, Davidson T, Filippova A, Gilroy C, Goode BJ, Jahani E, Kashyap R, Kirchner A, McKay S, Morgan AC, Pentland A, Polimis K, Raes L, Rigobon DE, Roberts CV, Stanescu DM, Suhara Y, Usmani A, Wang EH, Adem M, Alhajri A, AlShebli B, Amin R, Amos RB, Argyle LP, Baer-Bositis L, Büchi M, Chung B-R, Eggert W, Faletto G, Fan Z, Freese J, Gadgil T, Gagné J, Gao Y, Halpern-Manners A, Hashim SP, Hausen S, He G, Higuera K, Hogan B, Horwitz IM, Hummel LM, Jain N, Jin K, Jurgens D, Kaminski P, Karapetyan A, Kim EH, Leizman B, Liu N, Möser M, Mack AE, Mahajan M, Mandell N, Marahrens H, Mercado-Garcia D, Mocz V, Mueller-Gastell K, Musse A, Niu Q, Nowak W, Omidvar H, Or A, Ouyang K, Pinto KM, Porter E, Porter KE, Qian C, Rauf T, Sargsyan A, Schaffner T, Schnabel L, Schonfeld B, Sender B, Tang JD, Tsurkov E, Loon A van, Varol O, Wang X, Wang Z, Wang J, Wang F, Weissman S, Whitaker K, Wolters MK, Woon WL, Wu J, Wu C, Yang K, Yin J, Zhao B, Zhu C, Brooks-Gunn J, Engelhardt BE, Hardt M, Knox D, Levy K, 
Narayanan A, Stewart BM, Watts DJ, McLanahan S. Measuring the predictability of life outcomes with a scientific mass collaboration. Proc Natl Acad Sci. 2020 Apr 14;117(15):8398-403.

12. Silberzahn R, Uhlmann EL, Martin DP, Anselmi P, Aust F, Awtrey E, Bahník Š, Bai F, Bannard C, Bonnier E, Carlsson R, Cheung F, Christensen G, Clay R, Craig MA, Dalla Rosa A, Dam L, Evans MH, Flores Cervantes I, Fong N, Gamez-Djokic M, Glenz A, Gordon-McKeon S, Heaton TJ, Hederos K, Heene M, Hofelich Mohr AJ, Högden F, Hui K, Johannesson M, Kalodimos J, Kaszubowski E, Kennedy DM, Lei R, Lindsay TA, Liverani S, Madan CR, Molden D, Molleman E, Morey RD, Mulder LB, Nijstad BR, Pope NG, Pope B, Prenoveau JM, Rink F, Robusto E, Roderique H, Sandberg A, Schlüter E, Schönbrodt FD, Sherman MF, Sommer SA, Sotak K, Spain S, Spörlein C, Stafford T, Stefanutti L, Tauber S, Ullrich J, Vianello M, Wagenmakers E-J, Witkowiak M, Yoon S, Nosek BA. Many Analysts, One Data Set: Making Transparent How Variations in Analytic Choices Affect Results. Adv Methods Pract Psychol Sci. 2018 Sep $1 ; 1(3): 337-56$.

13. Botvinik-Nezer R, Holzmeister F, Camerer CF, Dreber A, Huber J, Johannesson M, Kirchler M, Iwanir R, Mumford JA, Adcock RA, Avesani P, Baczkowski BM, Bajracharya A, Bakst L, Ball S, Barilari M, Bault N, Beaton D, Beitner J, Benoit RG, Berkers RMWJ, Bhanji JP, Biswal BB, Bobadilla-Suarez S, Bortolini T, Bottenhorn KL, Bowring A, Braem S, Brooks HR, Brudner EG, Calderon CB, Camilleri JA, Castrellon JJ, Cecchetti L, Cieslik EC, Cole ZJ, Collignon O, Cox RW, Cunningham WA, Czoschke S, Dadi K, Davis CP, Luca AD, Delgado MR, Demetriou L, Dennison JB, Di X, Dickie EW, Dobryakova E, Donnat CL, Dukart J, Duncan NW, Durnez J, Eed A, Eickhoff SB, Erhart A, Fontanesi L, Fricke GM, Fu S, Galván A, Gau R, Genon S, Glatard T, Glerean E, Goeman JJ, Golowin SAE, González-García C, Gorgolewski KJ, Grady CL, Green MA, Guassi Moreira JF, Guest O, Hakimi S, Hamilton JP, Hancock R, Handjaras G, Harry BB, Hawco C, Herholz P, Herman G, Heunis S, Hoffstaedter F, Hogeveen J, Holmes S, Hu C-P, Huettel SA, Hughes ME, Iacovella V, Iordan AD, Isager PM, Isik AI, Jahn A, Johnson MR, Johnstone T, Joseph MJE, Juliano AC, Kable JW, Kassinopoulos M, Koba C, Kong X-Z, Koscik TR, Kucukboyaci NE, Kuhl BA, Kupek S, Laird AR, Lamm C, Langner R, Lauharatanahirun N, Lee H, Lee S, Leemans A, Leo A, Lesage E, Li F, Li MYC, Lim PC, Lintz EN, Liphardt SW, Losecaat Vermeer AB, Love BC, Mack ML, Malpica N, Marins T, Maumet C, McDonald K, McGuire JT, et al. Variability in the analysis of a single neuroimaging dataset by many teams. Nature. 2020 Jun;582(7810):84-8.

14. Dutilh G, Annis J, Brown SD, Cassey P, Evans NJ, Grasman RPPP, Hawkins GE, Heathcote A, Holmes WR, Krypotos A-M, Kupitz CN, Leite FP, Lerche V, Lin Y-S, Logan GD, Palmeri TJ, Starns JJ, Trueblood JS, van Maanen L, van Ravenzwaaij D, Vandekerckhove J, Visser I, Voss A, White CN, Wiecki TV, Rieskamp J, Donkin C. The Quality of Response Time Data Inference: A Blinded, Collaborative Assessment of the Validity of Cognitive Models. Psychon Bull Rev. 2019 Aug 1;26(4):1051-69.

15. Fillard P, Descoteaux M, Goh A, Gouttard S, Jeurissen B, Malcolm J, Ramirez-Manzanares A, Reisert M, Sakaie K, Tensaouti F, Yo T, Mangin J-F, Poupon C. Quantitative evaluation of 10 tractography algorithms on a realistic diffusion MR phantom. NeuroImage. 2011 May $1 ; 56(1): 220-34$.

16. Starns JJ, Cataldo AM, Rotello CM, Annis J, Aschenbrenner A, Bröder A, Cox G, Criss A, Curl RA, Dobbins IG. Assessing theoretical conclusions with blinded inference to investigate a potential inference crisis. Adv Methods Pract Psychol Sci. 2019;2(4):335-49.

17. Maier-Hein KH, Neher PF, Houde J-C, Côté M-A, Garyfallidis E, Zhong J, Chamberland M, Yeh F-C, Lin Y-C, Ji Q, Reddick WE, Glass JO, Chen DQ, Feng Y, Gao C, Wu Y, Ma J, He R, Li Q, Westin C-F, Deslauriers-Gauthier S, González JOO, Paquette M, St-Jean S, Girard G, Rheault F, Sidhu J, Tax CMW, Guo F, Mesri HY, Dávid S, Froeling M, Heemskerk AM, Leemans A, Boré A, Pinsard B, Bedetti C, Desrosiers M, Brambati S, Doyon J, Sarica A, Vasta R, Cerasa A, Quattrone A, Yeatman J, Khan AR, Hodges W, Alexander S, Romascano D, Barakovic M, Auría A, Esteban O, Lemkaddem A, Thiran J-P, Cetingul HE, Odry BL, Mailhe B, Nadar MS, Pizzagalli F, Prasad G, Villalon-Reina JE, Galvis J, Thompson PM, Requejo FDS, Laguna PL, Lacerda LM, Barrett R, Dell'Acqua F, Catani M, Petit L, Caruyer E, Daducci A, Dyrby TB, Holland-Letz T, Hilgetag CC, Stieltjes B, Descoteaux M. The challenge of mapping the human 
connectome based on diffusion tractography. Nat Commun [Internet]. 2017 Nov 7 [cited 2021 Mar 18];8. Available from: https://www.ncbi.nlm.nih.gov/pmc/articles/PMC5677006/

18. Poline J-B, Strother SC, Dehaene-Lambertz G, Egan GF, Lancaster JL. Motivation and synthesis of the FIAC experiment: Reproducibility of fMRI results across expert analyses. Hum Brain Mapp. 2006;27(5):351-9.

19. Rawlinson HS, Talbot F, Hincks E, Oppert J. Inscription of Tiglath Pileser I, King of Assyria, BC 1150, as translated by H. Rawlinson Fox Talbot Dr Hincks Dr Oppert Publ R Asiat Soc Lond JW Park Son. 1857;

20. Wilkinson MD, Dumontier M, Aalbersberg IjJ, Appleton G, Axton M, Baak A, Blomberg N, Boiten J-W, da Silva Santos LB, Bourne PE, Bouwman J, Brookes AJ, Clark T, Crosas M, Dillo I, Dumon O, Edmunds S, Evelo CT, Finkers R, Gonzalez-Beltran A, Gray AJG, Groth P, Goble C, Grethe JS, Heringa J, 't Hoen PAC, Hooft R, Kuhn T, Kok R, Kok J, Lusher SJ, Martone ME, Mons A, Packer AL, Persson B, Rocca-Serra P, Roos M, van Schaik R, Sansone S-A, Schultes E, Sengstag T, Slater T, Strawn G, Swertz MA, Thompson M, van der Lei J, van Mulligen E, Velterop J, Waagmeester A, Wittenburg P, Wolstencroft K, Zhao J, Mons B. The FAIR Guiding Principles for scientific data management and stewardship. Sci Data. 2016 Mar 15;3:160018.

21. Aczel B, Szaszi B, Sarafoglou A, Kekecs Z, Kucharský Š, Benjamin D, Chambers CD, Fisher A, Gelman A, Gernsbacher MA, Ioannidis JP, Johnson E, Jonas K, Kousta S, Lilienfeld SO, Lindsay DS, Morey CC, Munafò M, Newell BR, Pashler H, Shanks DR, Simons DJ, Wicherts JM, Albarracin D, Anderson ND, Antonakis J, Arkes HR, Back MD, Banks GC, Beevers C, Bennett AA, Bleidorn W, Boyer TW, Cacciari C, Carter AS, Cesario J, Clifton C, Conroy RM, Cortese M, Cosci F, Cowan N, Crawford J, Crone EA, Curtin J, Engle R, Farrell S, Fearon P, Fichman M, Frankenhuis W, Freund AM, Gaskell MG, Giner-Sorolla R, Green DP, Greene RL, Harlow LL, de la Guardia FH, Isaacowitz D, Kolodner J, Lieberman D, Logan GD, Mendes WB, Moersdorf L, Nyhan B, Pollack J, Sullivan C, Vazire S, Wagenmakers E-J. A consensus-based transparency checklist. Nat Hum Behav. 2020 Jan;4(1):4-6.

22. Lundberg I, Narayanan A, Levy K, Salganik MJ. Privacy, Ethics, and Data Access: A Case Study of the Fragile Families Challenge. Socius. 2019 Jan 1;5:2378023118813023.

23. Drechsler J. Synthetic datasets for statistical disclosure control: theory and implementation. Vol. 201. Springer Science \& Business Media; 2011.

24. Quintana DS. A synthetic dataset primer for the biobehavioural sciences to promote reproducibility and hypothesis generation. Zaidi M, Büchel C, Bishop DVM, editors. eLife. 2020 Mar 11;9:e53275.

25. Kindel AT, Bansal V, Catena KD, Hartshorne TH, Jaeger K, Koffman D, McLanahan S, Phillips M, Rouhani S, Vinh R. Improving metadata infrastructure for complex surveys: Insights from the Fragile Families Challenge. Socius. 2019;5:2378023118817378.

26. Schweinsberg M, Feldman M, Staub N, van den Akker OR, van Aert RCM, van Assen MALM, Liu Y, Althoff T, Heer J, Kale A, Mohamed Z, Amireh H, Venkatesh Prasad V, Bernstein A, Robinson E, Snellman K, Amy Sommer S, Otner SMG, Robinson D, Madan N, Silberzahn R, Goldstein P, Tierney W, Murase T, Mandl B, Viganola D, Strobl C, Schaumans CBC, Kelchtermans S, Naseeb C, Mason Garrison S, Yarkoni T, Richard Chan CS, Adie P, Alaburda P, Albers C, Alspaugh S, Alstott J, Nelson AA, Ariño de la Rubia E, Arzi A, Bahník Š, Baik J, Winther Balling L, Banker S, AA Baranger D, Barr DJ, Barros-Rivera B, Bauer M, Blaise E, Boelen L, Bohle Carbonell K, Briers RA, Burkhard O, Canela M-A, Castrillo L, Catlett T, Chen O, Clark M, Cohn B, Coppock A, Cugueró-Escofet N, Curran PG, Cyrus-Lai W, Dai D, Valentino Dalla Riva G, Danielsson H, Russo R de FSM, de Silva N, Derungs C, Dondelinger F, Duarte de Souza C, Tyson Dube B, Dubova M, Mark Dunn B, Adriaan Edelsbrunner P, Finley S, Fox N, Gnambs T, Gong Y, Grand E, Greenawalt B, Han D, Hanel PHP, Hong AB, Hood D, Hsueh J, Huang L, Hui KN, Hultman KA, Javaid A, Ji Jiang L, Jong J, Kamdar J, Kane D, Kappler G, Kaszubowski E, Kavanagh CM, Khabsa M, Kleinberg B, Kouros J, Krause H, Krypotos A-M, Lavbič D, Ling Lee R, Leffel T, Yang Lim W, Liverani S, Loh B, Lønsmann D, Wei Low J, Lu A, MacDonald K, Madan CR, Hjorth Madsen L, Maimone C, Mangold A, Marshall A, Ester Matskewich H, Mavon K, McLain KL, McNamara AA, McNeill M, Mertens U, Miller D, Moore $\mathrm{B}$, et al. Same data, different conclusions: Radical dispersion in empirical results when independent analysts operationalize and test the same hypothesis. Organ Behav Hum Decis 
Process. 2021 Jul 1;165:228-49.

27. Chambers CD. Registered reports: a new publishing initiative at Cortex. Cortex. 2013;49(3):609-10.

28. Gøtzsche PC. Blinding during data analysis and writing of manuscripts. Control Clin Trials. 1996 Aug; 17(4):285-90; discussion 290-293.

29. Boettiger C. An introduction to Docker for reproducible research. ACM SIGOPS Oper Syst Rev. 2015 Jan 20;49(1):71-9.

30. Nüst D, Sochat V, Marwick B, Eglen SJ, Head T, Hirst T, Evans BD. Ten simple rules for writing Dockerfiles for reproducible data science. PLOS Comput Biol. 2020 Nov 10;16(11):e1008316.

31. Liu DM, Salganik MJ. Successes and Struggles with Computational Reproducibility: Lessons from the Fragile Families Challenge. Socius. 2019 Jan 1;5:2378023119849803.

32. Hurlin C, Perignon C. Reproducibility Certification in Economics Research [Internet]. Rochester, NY: Social Science Research Network; 2019 Jul [cited 2021 Jan 13]. Report No.: ID 3418896. Available from: https://papers.ssrn.com/abstract=3418896

33. Pérignon C, Gadouche K, Hurlin C, Silberman R, Debonnel E. Certify reproducibility with confidential data. Science. 2019 Jul 12;365(6449):127-8.

34. Holcombe AO, Kovacs M, Aust F, Aczel B. Tenzing: documenting contributorship using CRediT. 2020;

35. Simonsohn U, Simmons JP, Nelson LD. Specification curve analysis. Nat Hum Behav. 2020;4(11):1208-14.

36. Schönbrodt F, Patel CJ, Ioannidis J, Boulesteix A-L, Hoffmann S. Comparing the vibration of effects due to model, data pre-processing and sampling uncertainty on a large data set in personality psychology.

37. Liu Y, Kale A, Althoff T, Heer J. Boba: Authoring and visualizing multiverse analyses. IEEE Trans Vis Comput Graph. 2020;27(2):1753-63.

38. Dragicevic P, Jansen Y, Sarma A, Kay M, Chevalier F. Increasing the transparency of research papers with explorable multiverse analyses. In: Proceedings of the 2019 CHI Conference on Human Factors in Computing Systems. 2019. p. 1-15.

39. Hoeting JA, Madigan D, Raftery AE, Volinsky CT. Bayesian model averaging: a tutorial (with comments by M. Clyde, David Draper and EI George, and a rejoinder by the authors. Stat Sci. 1999;14(4):382-417.

40. Richardson S, Gilks WR. A Bayesian approach to measurement error problems in epidemiology using conditional independence models. Am J Epidemiol. 1993;138(6):430-42.

Acknowledgements: This research was not funded. AS was supported by a talent grant from the Netherlands Organisation for Scientific Research (NWO) to AS (406-17-568). RB-N is an Awardee of the Weizmann Institute of Science - Israel National Postdoctoral Award Program for Advancing Women in Science. BAN was supported by grants from the John Templeton Foundation, Templeton World Charity Foundation, Templeton Religion Trust, and Arnold Ventures. SSt-J is supported by the Natural Sciences and Engineering Research Council of Canada (NSERC) [funding reference number BP-546283-2020] and the Fonds de recherche du Québec - Nature et technologies (FRQNT) [Dossier 290978]. JMW and ORvdA were supported by a Consolidator Grant (IMPROVE) from the European Research Council (ERC; grant no. 726361). YKK was supported by a grant from the European Research Council (ERC) under the European Union's Horizon 2020 research and innovation programme (ERC-CoG-2015; No 681466 to M Wichers). DvR was supported by a Dutch scientific 
organization VIDI fellowship grant (016.Vidi.188.001). LFB was supported by a Dutch scientific organization VENI fellowship grant (Veni 191G.037). MJS was supported by the US National Science Foundation (1760052).

Author contributors: BA and BS are joined first authors and guarantors. BA, BS, GN, and E-JW were responsible for the study conception and design. ORvdA, CJA, MALMvA, JAB, DB, UB, RB-N, LFB, NB, EC, AMC, NC, A Delios, NNNvD, CD, JBvD, A Dreber, GD, GFE, MAG, RH, SH, FH, JH, MJ, KJJ, ATK, MK, YKK, DSL, J-FM, DM, MRM, BRN, BAN, RAP, DvR, JR, MJS, AS, TS, MS, DS, RS, DJS, BAS, SSt-J, JJS, ELU, and JW served as expert panel in the development of the guidance and checklist. All authors participated in drafting and critically revising the manuscript. The corresponding author attests that all listed authors meet authorship criteria and that no others meeting the criteria have been omitted.

Competing interests: We have read the journal's policy and the authors of this manuscript have the following competing interests: B.A.N. is Executive Director of the Center for Open Science, a non-profit technology and culture change organization with a mission to increase openness, integrity, and reproducibility of research. The other authors declare no competing interest.

Transparency declaration: The lead author affirms that the manuscript is an honest, accurate, and transparent account of the work being reported; that no important aspects of the study have been omitted; and that any discrepancies from the work as planned have been explained.

Data and materials availability: All anonymized data as well as the survey materials are publicly shared on the Open Science Framework page of the project: https://osf.io/4zvst/. Our methodology and data-analysis plan were preregistered. The preregistration document can be accessed at: https://osf.io/dgrua.

\section{Appendices:}

Appendix 1 - Reporting Template for Multi-Analyst Studies Appendix 2 - Reporting Checklist for Multi-Analyst Studies 
Appendix 1 - Reporting Template for Multi-Analyst Studies

The following template was created to support the reporting of multi-analyst projects. The present document focuses on methods and details of the multi-analysis process itself.

As multi-analyst projects can differ along many dimensions, this template should be freely modified according to the parameters of the specific projects. Furthermore, this document could also serve as a checklist on what to include in the methods section of multi-analyst projects.

\section{Reporting Template}

We aimed to recruit a minimum number of in order to check the robustness of the conclusions / assess variability of analyses.

Co-analysts were recruited using the following sources:

The eligibility criteria for our co-analysts were the following:

In total, __ co-analysts signed up to the project, of whom met the eligibility criteria. of the co-analysts came from Source 1 , from Source 2 ...

The lead team also conducted / did not conduct an independent analysis on the same research question as the co-analysts.

After the recruitment, the co-analysts were informed about the following:

(a) their tasks and responsibilities;

(b) the project code of conduct (e.g., confidentiality/ non-disclosure agreements);

(c) the plans for publishing the research report and presenting the data, analyses, and conclusion;

(d) the conditions for an analysis to be included or excluded from the study;

(e) whether their names will be publicly linked to the analyses;

(f) the co-analysts' opportunities to update or revise their analyses;

(g) the project time schedule;

(h) the nature and criteria of compensation (e.g., authorship, monetary rewards, etc.);

(i) why and at what stage co-analysts are allowed to communicate about the analyses (e.g., to catch errors or call attention to outlying data points).

In order to ensure independence, all members of the co-analyst teams signed an agreement/agreed that they would not publicize, release, or discuss their analysis choices and the results with anyone until after all initial reports have been submitted. The non-disclosure agreement is available at 
After accepting the conditions of participation, co-analysts were provided with the research questions and the dataset(s) accompanied with a codebook that contained a comprehensive explanation of the variables and the datafile structure on $20_{-}$- _ _ _ . The research questions were provided without communicating any preferred analysis choices and or expectations about the conclusions. The dataset along with the accompanying codebook is available at To ensure that the co-analysts understand the restrictions on the use of the data, including issues of ethics, privacy, confidentiality, or ownership, we

The deadline of the co-analysts to submit their analysis code with explanatory comments (or a detailed description of their point-and-click analyses), their conclusions, and an explanation of how their conclusions follow from their results has been 20

Out of the participating teams, submitted their work by this deadline. An additional teams submitted their work by an extended deadline of

Out of these submitting teams, teams consisted of members, teams consisted of members...

Out of the _ co-analysts, there were senior researchers, post-doctoral researchers, graduate students, and members from other positions.

Out of the __ submitted analyses, __ were withdrawn, and were omitted from the summary analysis for the following reasons: ...

From _ co-analyst teams who submitted their work, we received analyses and results. The lead team made the commented code, results, and conclusions of all non-withdrawn analyses publicly available before / at the time of submitting the research report.

The submitted code, results, and conclusions were processed the following way:

The summary of the results of the co-analysts are:

The limitations and potential biases of the study are as follows:

[A practical way to summarize the analytic approaches and results is to present them in a table or graph. Before approving the publication of the study, the co-analysts could add their reflections on the project or its results. These comments can supplement the report of the study.

Some previous examples for summarizing and visualizing the results are here and here.] 


\section{Appendix 2 - Reporting Checklist for Multi-Analyst Studies}

\section{Reporting Checklist for Multi-Analyst Studies}

\begin{tabular}{|c|l|l|}
\hline $\begin{array}{c}\text { Item } \\
\text { No }\end{array}$ & Recommended reporting item & $\begin{array}{l}\text { Page number(s) } \\
\text { where item is } \\
\text { reported }\end{array}$ \\
\hline 1 & Justification for the number of co-analysts & \\
\hline 2 & Eligibility criteria and recruitment of co-analysts & \\
\hline 3 & How co-analysts were given the data sets and research questions & \\
\hline 4 & How the independence of analyses was ensured & \\
\hline 5 & Numbers of and reasons for withdrawals and omissions of analyses & \\
\hline 6 & Whether the lead team conducted an independent analysis & \\
\hline 7 & How the results were processed & \\
\hline 8 & Summary of the results of co-analysts & \\
\hline 9 & Limitations and potential biases of the study & \\
\hline 10 & How the reader can access the data and code for all analyses & \\
\hline
\end{tabular}

